\title{
ANNOUNCEMENT
}

\section{Call for Papers: Special Issue on Social and Technical Trade-Offs}

Guest Editors: Solon Barocas, PhD, danah boyd, PhD, Sorelle Friedler, PhD, and Hanna Wallach, PhD

\section{Deadline for submission: September 15, 2016}

This special issue on Social and Technical Trade-Offs aims to serve two main purposes:

1. To highlight exciting and novel work in machine learning, artificial intelligence, data mining, and data science that articulates, examines, challenges, and addresses the technical and social trade-offs involved in the analysis and interpretation of big data.

2. To pose practical, grounded, and socially-oriented challenges for researchers in machine learning, artificial intelligence, data mining, and data science to motivate and guide their research.

Working with "big data" isn't easy, especially when it involves social data. Researchers and practitioners must make hard choices when cleaning and processing data, grapple with biased data sets and missing data, and evaluate the social and technical trade-offs involved in analysis and interpretation. What are the ethical implications of these choices? What happens when we get it wrong? How can we prioritize reproducibility? What happens when biased data and imperfect methods are combined in unexpected ways? This special issue will examine the trade-offs that emerge from the interconnected nature of the social and technical decision-making that lies at the heart of big data.

We encourage submissions that focus on challenges and questions involving large-scale social data, and that are deployed (or are in the process of being deployed) in the real world.

Area of focus include (but are not limited to):

- Surveillance and privacy

- Healthcare, medicine, and public health

- Criminal justice and policing

- Education and learning

- Disaster relief

- Urban planning, housing, and infrastructure

- Finance, scoring, and insurance

- Public administration and public policy

- Autonomous experimentation

- Targeted advertising

Example questions that are relevant include (but are not limited to):

- How should we strike a balance between model performance and interpretability?

- How can we formalize social concepts in ways that are amenable to machine learning methods? How do these formalizations influence the choice of machine learning method? 
- How does uncertainty and noise inherent to real-world data sets affect the use of these data sets and the use of results obtained from them via machine learning methods?

- How can we incorporate social and ethical considerations into our validation methods and choices? What are the social costs of errors or class imbalance and the distribution of those errors across populations? What are the social implications of prioritizing false positive rates vs. false negative rates?

- When is it appropriate to collect additional data about minority or underrepresented populations? How should we address the need for balanced data sets without imposing a "diversity tax?" How should we weigh the social and financial associated costs and benefits?

- What are the social consequences and tradeoffs involved in feature selection?

We encourage submissions from organizations that may do not typically write research papers. In addition to submissions from universities and corporations, we welcome submissions from government agencies, nonprofit organizations, startups, and foundations.

These submissions might be:

- Papers that describe and evaluate new and/or existing methods that balance social and technical factors in decision-making using or surrounding big data.

- Papers that describe trade-offs that emerged during the design and implementation of big data systems in industry, government, or nonprofit settings.

- Position papers that highlight sociotechnical challenges that need to be overcome in order to make methods that are suited to responsibly solving large-scale social challenges.

Big Data is a highly innovative, peer-reviewed journal that provides a unique forum for worldclass research exploring the challenges and opportunities in collecting, analyzing, and disseminating vast amounts of data, including data science, big data infrastructure and analytics, and pervasive computing. There are many advantages to publishing in Big Data, including:

- Indexed in Thomson Reuters Web of Science ${ }^{\mathrm{TM}}$ Science Citation Index Expanded (SciSearch) and Journal Citation Reports/Science Edition

- Fast and user-friendly electronic submission

- Rapid, high-quality peer review

- Maximum exposure: accessible in 170 countries worldwide

\section{Editorial questions?}

Please address any questions to bd-tradeoffs@lists.datasociety.net

Deadline for manuscript submission: September 15, 2016. Please submit your papers online to our web-based manuscript submission and peer-review at: www.liebertpub.com/manuscript/big

Visit our website to read about the Journal and to view a sample issue: www.liebertpub.com/big

\section{Manuscript preparation or technical questions?}

Contact authorservices@liebertpub.com

Cite this article as: Barocas B, boyd d, Friedler S, Wallach H (2016) Call

for papers: Special issue on social and technical trade-offs. Big Data

4:1, 3-4, DOI: 10.1089/big.2016.29006.cfps. 